\title{
Cosmic neutrinos
}

\section{Teresa MONTARULI*†}

DPNC, Université de Genève, 1211 Genève, Suisse

E-mail: teresa.montarulieunige.ch

The hunt for cosmic neutrinos has effectively just started. Recently, large detectors have been completed and are running, such as IceCube at the South Pole and ANTARES in the Mediterranean sea. Such detectors begin to access the region where neutrino fluxes at energies above $100 \mathrm{GeV}$ are expected from shock and jet acceleration processes in the universe. The expectation for these fluxes comes from common knowledge on particle interactions and from the observation of cosmic rays. As a matter of fact, the sources that accelerate cosmic rays are also the powerful engines that would produce neutrinos. How efficiently this production occurs depends on the nature of sources and of their surrounding environments. The non observation of cosmic events is on one side a source of disappointment, on the other side it represents by itself an important result. If seen in the context of a multi-messenger science, the combination of photon and cosmic ray experiment results brings invaluable information.

The 2011 Europhysics Conference on High Energy Physics-HEP 2011,

July 21-27, 2011

Grenoble, Rhône-Alpes France

* Speaker.

${ }^{\dagger}$ At the time of the conference at University of Wisconsin - Madison. 


\section{Introduction on neutrino telescopes}

Since the times they were discovered at the beginning of last century, cosmic rays (CRs) have been carriers of large amount of information concerning particle physics, plasma physics and astrophysics. Despite the relevant amount of measurements we gathered on their spectrum and composition, we did not yet observe the sites where these particles are created in the Galaxy and outside it. The study of the universe through CRs is a major topic of 'Astroparticle Physics'. This field joins the community of physicists and astronomers and fascinates people and researches for the diversity of physics items and for the unusual locations where detectors are built. Such physics items concern fundamental questions on the universe evolution, its constituents, particle interactions, verification of general relativity, and life science. I will focus here on some selected topics concerning the search of cosmic sources in Cherenkov neutrino telescopes, large extensive air shower arrays having being covered elsewhere at this conference [1]. Indirect dark matter detection in IceCube and ANTARES were covered at this conference in [2,3]. This topic is of high interest also in view of the fact that current LHC limits are pushing new physics in the high energy region, where indirect detection is competitive with respect to direct measurements.

The South Pole, where the first cubic-kilometer telescope IceCube is located, is a special observation site since the same view of the sky can be enjoyed at all hours. IceCube was completed during the austral summer 2010-11 and measures since April 2011 a muon rate of $\sim 2.7 \mathrm{kHz}$. At the time of writing, fall 2011, the running lifetime is $99 \%$. The completion of IceCube on schedule [4] and the operation of ANTARES in the Mediterranean sea in its final configuration since May 2008 [5] are two important milestones for neutrino astronomy. Currently, there is high momentum in the European community for the construction of a detector in the Mediterranean sea of better sensitivity than IceCube for galactic sources [6]. The IceCube Observatory is an ensemble of detectors composed by: a cubic-kilometer neutrino telescope deep in the ice sensitive to neutrinos with $E_{v} \gtrsim 100 \mathrm{GeV}$; the extensive air shower array IceTop and the recently added DeepCore [7], a denser array with the aim of improving the performance at energies $\lesssim 1 \mathrm{TeV}$ for dark matter, neutrino oscillation and SN collapse neutrino searches. The deep ice hosts 5160 optical modules, glass spheres enclosing 10-inch photomultipliers (PMTs) and associated electronics for waveform digitization. These Digital Optical Modules (DOMs) are autonomous small computers that communicate with the surface laboratory. The time and the amount of photons that reach the ns-precision DOMs make the reconstruction of particle direction and energy possible. After almost 7 years from the installation of the first string, $98.5 \%$ of the DOMs are in stable operation. IceTop is composed of stations of 2 tanks of frozen water seen by 2 DOMs separated by about $10 \mathrm{~m}$ at the top of each string. It can be used in coincidence with the deep-ice detector for CR composition studies, angular and energy reconstruction cross calibrations and to veto background muons produced in atmospheric showers. A coincident CR induced event through IceTop and IceCube is shown in Fig. 1 (left).

Occasionally, neutrinos interact with matter in the proximity of the detector or inside its instrumented volume. The Earth can be used as a filter to reduce the main background to the neutrino measurement: atmospheric muons. Upgoing muons are a signature of neutrinos since they are the only particles that can cross the entire Earth. Since the neutrino cross section increases almost linearly with energy, at energies above $10^{5} \mathrm{GeV}$ the interaction length becomes comparable to the 
Earth diameter. Hence, only downgoing or horizontal ultra-high energy neutrinos can be detected. At analysis level atmospheric neutrinos contribute about 200 upgoing neutrino events per day in the full IceCube. A muon neutrino charged current interaction produces a muon that propagates through the detector for a distance that depends on energy. A neutrino-induced upgoing muon event releasing about $10 \mathrm{TeV}$ in the detector and a $50 \mathrm{TeV}$ candidate electron neutrino cascade are shown in Fig. 1 (left). Electron and tau charged current interactions and all-flavor neutrino neutral current interactions produce cascade events that have an approximately isotropic distribution of light and are 'point-like' on the scale of the distance between PMTs. Light propagates from the interaction vertex up to some hundreds of meters. The $1.5 \mathrm{~km}$ layer of ice above IceCube reduces the atmospheric muon flux by about 4 orders of magnitude. Further reduction of 2 orders of magnitude can be obtained using the 3 external rings of strings of IceCube that surround DeepCore and 40 horizontal layers of DOMs above it as veto. Since the ratio between the atmospheric muon and neutrino fluxes at trigger level in IceCube is about $10^{6}$, the veto is effective to reduce backgrounds to neutrino detection level and to identify neutrinos with vertex contained in the instrumented region [7]. Using this veto and additional containment and reconstruction quality cuts requirements allows the measurement of neutrinos produced inside this instrumented region and contained cascade events. Muon events are used for this searches, since their pointing accuracy is $\sim 1^{o}$ as shown in Fig. 1(right). The gain in sensitivity for point-source searches is directly proportional to the angular resolution squared in an almost background free regime, which applies to time dependent point-like source searches in IceCube. The pointing capabilities of IceCube are checked using the Moon shadow that is detected at the level of more than $13 \sigma$ with 59 strings of IceCube [8]. Muon energy is reconstructed thanks to the linear dependency of the muon energy losses in the regime where stochastic processes dominate over ionization $(\gtrsim 1 \mathrm{TeV})$. The achieved resolution is of the order of $0.15-0.2$ in $\log 10$ of the muon energy between $1 \mathrm{TeV}$ up to about $10^{9} \mathrm{GeV}$.

\section{Discussion on current results for extragalactic and galactic sources}

An astronomical messenger has to point back to its cosmic source that sent it to us. Neutrinos are neutral and so undeflected by magnetic fields in the Galaxy and in the intergalactic space. Potentially, they are the most sensitive messengers because they are weekly interacting particles and hence they probe the interior of sources and reach us from cosmological distances. As illustrated by the well known Hillas' diagram [9], the maximum energy of an accelerated particle of charge $Z e$ depends on the size of the accelerating region and on the magnetic field: $E_{\max } \sim \Gamma Z B R$, where $\Gamma$ is the jet Lorenz factor. This simple relationship is obtained from the requirement that the Larmour radius of accelerated particles is smaller than the acceleration region, but considerations on energy losses and on the age of the accelerating process have to be accounted for. For instance, gamma-ray bursts (GRBs) are thought to be efficient accelerators of ultra-high energy cosmic rays (UHECR). The maximum acceleration energy is compatible with the highest energy events observed by giant extensive air shower arrays of $10^{21} \mathrm{eV}$, such as HiReS and Auger (for a review see [10]). This has been demonstrated imposing that the duration of the burst is larger than the acceleration time $\frac{R}{\Gamma c} \geq \frac{r_{L}}{c}$ and that the energy gained in the acceleration process is larger than the synchrotron energy $\operatorname{loss}\left(t_{s y n} \geq t_{a c c}\right)[11,12]$. 

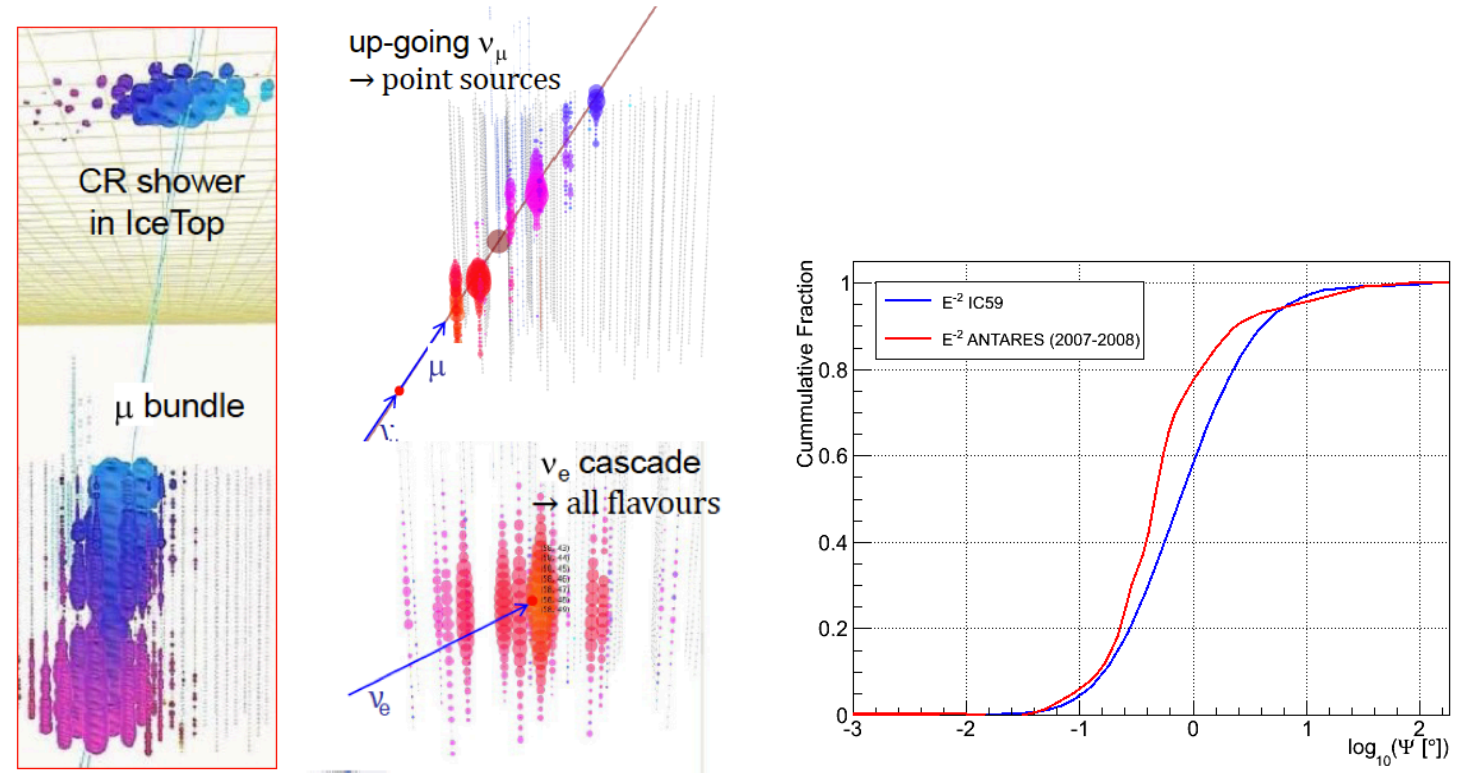

Figure 1: Left: An event detected by IceTop and IceCube in coincidence and two high energy muon and electron neutrino events. The color code (from red to blue) is proportional to the DOM hit time and the DOM size is proportional to the deposited charge. Right: Comparison of the point spread function (PSF) of ANTARES and IceCube-59 strings for muon neutrino events: fraction of events inside the $\log 10$ of the angle between the true neutrino and the reconstructed secondary muon for an $E^{-2}$ neutrino spectrum. For ANTARES (IceCube-59) $60 \%$ of the events are inside an angle of $0.6^{\circ}\left(1^{\circ}\right)$ from a source.

Candidate extragalactic sources of UHECR are GRBs and black hole jets in Active Galactic Nuclei (AGN). Not only they are efficient accelerators but they also have the right density distribution and power per source that is needed to explain the spectrum of UHECRs. As a matter of fact, the spectrum and composition of CRs contains information on the nature of cosmic accelerators. The all-particle CR flux falls as an approximate power-law in energy with two evident changes of slope: the knee at $\sim Z \times$ few $\mathrm{PeV}$ ( $\mathrm{Z}$ is the charge of nuclei) and the ankle at about $E \sim 4 \times 10^{9} \mathrm{GeV}$. Since the most powerful engines of the universe host intense magnetic fields, they can be 'optically thick'. In these sources, protons and nuclei are accelerated and interact in radiation fields and matter in the source but only neutrinos can emerge. On the other hand, in 'optically thin' sources neutrons produced in proton interactions can escape the magnetic fields and produce both the cosmic rays that we observe in large extensive air shower arrays and the neutrinos observed by neutrino telescopes. Hence, there is an intimate relationship between the energy we observe in the UHECR spectrum and expected neutrino fluxes. From this reasoning, Waxman \& Bahcall (W\&B) derived an upper limit on the diffuse neutrino emission from extragalactic optically-thin sources from which CRs escape [11]. This upper limit is estimated in the assumption that UHECRs are protons with $E^{-2}$ injection spectrum and is normalized on the UHECR measured in large extensive air shower arrays. The CR production rate used in Ref. [11] of $E^{2} d N / d E=10^{44}$ erg $\mathrm{Mpc}^{-3} \mathrm{yr}^{-1}$ was recently updated due to the results of HiReS and Auger that superseded the originally used AGASA data. This produced a decrease of a factor of about 2 in the normalization [12] to which another factor of 2 of reduction has to be added due to astrophysical neutrino oscil- 
lations. The resulting bound is about $10^{-8} \mathrm{GeV} \mathrm{cm}^{-2} \mathrm{~s}^{-1} \mathrm{sr}^{-1}$. This normalization procedure is affected by the indetermination of the energy at which extragalactic CRs begin to dominate over galactic ones between $10^{17}-10^{19} \mathrm{eV}$. Moreover, the resulting neutrino flux depends on the composition of extragalactic CRs, on the maximum energy of the primary injection spectrum and on the assumed cosmological source evolution. In Fig. 2 (left) the grey band indicates the region between the revised W\&B upper limit [12] to predictions for GRBs [12]. It can be seen that the 90\%CL upper limit of $1 / 2$ of the full IceCube begins to explore the relevant region for steady emissions of optically-thin sources.

Recent results on GRBs using neutrino-induced muon events for the 40-string configuration were published in [14] and 59 string data were combined providing considerably better limits discussed at this conference in [15]. A total of about $300 \mathrm{GRBs}$ were searched for neutrino emission with complementary analyses that did not find evidence for a signal. In the absence of a signal, the limits are combined and are a factor 0.22 of the model in [16] that consider neutrino fluxes for specific bursts. The model in [11], that applies to the average of BATSE GRBs, predicts 14.2 events. Models and upper limits are shown in Fig. 2 (right). Another model independent time scan, performed over time scales between $\pm 10 \mathrm{~s}$ and \pm 1 day with looser cuts, would have found 14.5 [16] and 27.7 [11] and measured no events.

The impact of the upper limit of IceCube on fireball predictions is discussed in [15]. For the case in which GRBs are the dominant sources of CRs, neutrino fluxes can be lowered by reducing the proton content below the level required to explain the CR spectrum, or the threshold for pion production can be increased by increasing the boost factor of the shocks, hence reducing the efficiency of neutrino production. It should be noted that primary protons may not escape the intense magnetic fields in the fireball. Hence, the CRs observed by giant extensive air showers could originate from the decay of secondary neutrons from $p \gamma \rightarrow \Delta^{+} \rightarrow n \pi^{+}$that can escape Bfields. In this second case, the W\&B upper limit would be higher by about a factor of 3-6 since the CR flux is normalized on secondary and not primary nucleons.

If GRBs were the sources of CRs, UHECRs would be proton dominated since nuclei would not survive in the fireballs. No correlation between UHECRs and neutrino events could be observed since neutrinos cannot arrive in time-coincidence with protons that would take a much longer time to reach us. IceCube data provide no evidence for such correlation [8]. The composition measurement and the observation of anisotropies for UHECR and the detection of UHE neutrinos would help understanding the nature of extragalactic sources. Pure proton or pure iron composition would produce similar spectral shape in the GZK cut-off region. Nonetheless, if iron dominates, expected neutrino fluxes from iron photo-disintegration would be orders of magnitude smaller than for pure proton composition and could be unaccessible to current detectors [10]. Moreover, if the composition would be dominated by high mass elements anisotropies become improbable unless B-fields are much lower than what we believe. The EHE search in IceCube for a diffuse flux of neutrinos selects downgoing and horizontal events with energies $\gtrsim 10^{6} \mathrm{GeV}$ based on the charge released in the detector as a function of the zenith angle [17]. No event survived the analysis cuts for a livetime of $333.5 \mathrm{~d}$ of the 40 string configuration and upper limits are shown in Fig. 3 (right) together with some models (references in [17]). The upper limit to the neutrino flux from optically thin sources [11] modified to include oscillations and including evolution of sources corresponds to 4.5 neutrino events/yr in 40 strings and 24.5 in 3 yrs of the full detector. The model GZK6, that includes the 

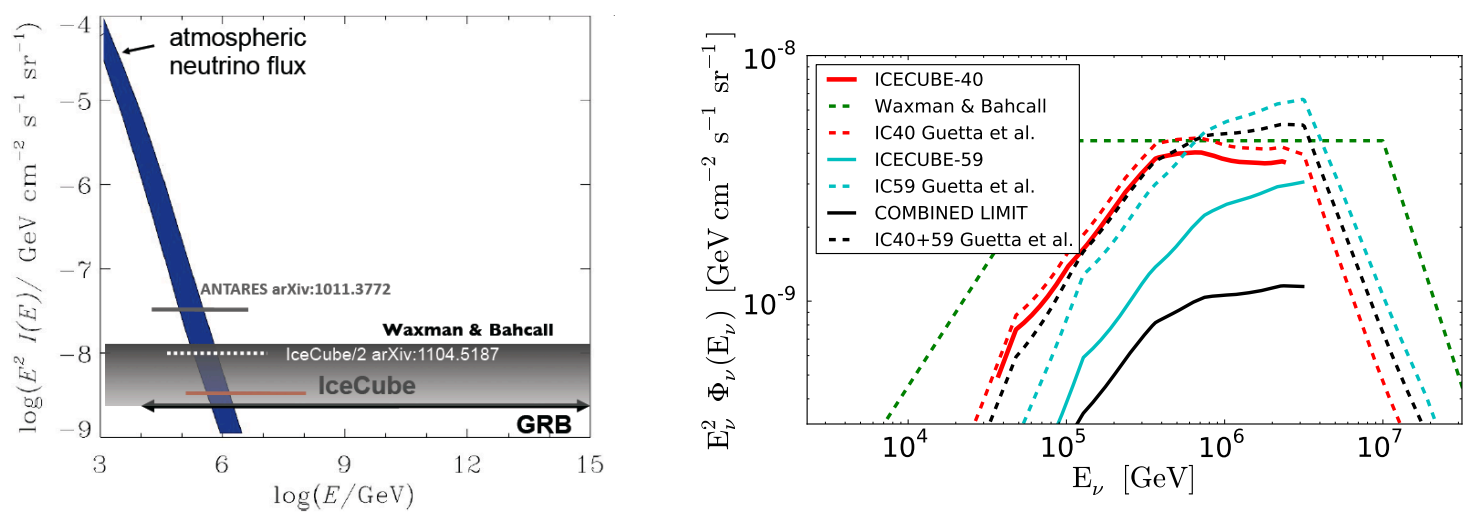

Figure 2: Left: $90 \%$ c.l. upper limits on the diffuse muon neutrino flux of $E^{-2}$ neutrinos for ANTARES [5] and 40-strings of IceCube [13] and a prediction for the full IceCube. The horizontal band represents the region between the revised W\&B upper limit and the region relevant for GRB fluxes and the oblique band atmospheric neutrinos. Right: The $90 \%$ c.l. upper limits on the muon neutrino flux from GRBs are shown with solid lines while models with dashed ones. Diffuse fluxes are obtained from fluences of all the considered bursts assuming a total of 667 uniformly distributed bursts per year. Two models are shown from [16] calculated for the bursts happened during the 40 and 59 string data takings. The W\&B prediction is shown too [11] (dashed curve that extends to lower energies). The lowest of the upper limits (solid black line) is the combined one for $40+59$ strings.

constraints from the Fermi-LAT diffuse gamma flux and the UHECR as measured by HiReS and Auger, would produce 4.8 events in 3 years of the full detector. A radio detector for ultra-high energy neutrinos $\left(\gtrsim 10^{17} \mathrm{eV}\right)$, ARA, is now under test and a proposal for a $100 \mathrm{~km}^{2}$ radio detector is submitted [18].

Other searches dedicated mainly to AGN flares have been developed in IceCube $[19,8]$ and ANTARES [5] to look for flares in coincidence with other X-ray or gamma detectors, such as Fermi and Imaging Cherenkov Telescopes. None found evidence of correlated neutrino emission. This 'triggered' searches reduce the trial factor by using X-ray and $\gamma$-ray information, but rely on the assumption that flares of neutrinos are in coincidence with flares in photons as a result of an enhanced acceleration power in the source. In order not to miss any signal from a flare if neutrinos are not in coincidence with photons, IceCube also adopt an untriggered approach looking for clusters of high energy neutrino events in space and time. For a neutrino flare of $1 \mathrm{~s}$ (1 day) the discovery potential is about a factor of 4 (3) better than for time-independent searches [20,8]. This analysis did not find a significant flare in the data taken with 40 strings [19]. The recently unblinded results with 59 strings of IceCube revealed a $1.4 \%$ significant flare of reconstructed duration of $\sim 10$ days with about 14 signal-like events that contributed to this significance. Given the large trial factor this flare did not reach the threshold required for discovery in IceCube of $5 \sigma$.

Time independent likelihood approaches have been used by ANTARES and IceCube [21, 20, 8] to detect the presence of a signal from any point in the sky among a large background of atmospheric neutrino upgoing events. In this region, the sensitivity for an $E^{-2}$ flux of neutrinos is peaked around some tens of $\mathrm{TeV}$. The background is mainly due to atmospheric neutrinos with a few percent contamination of mis-reconstructed atmospheric muons for IceCube and $40 \%$ for ANTARES. 
IceCube extended the search to the full sky including the southern sky that is dominated by downgoing atmospheric muons. A zenith-dependent energy cut selects high energy muons to reduce the high statistics that would prevent the application of the likelihood method. Hence, in the southern hemisphere IceCube sensitivity is peaked at higher energies between $\mathrm{PeV}$ and $\mathrm{EeV}$. The likelihood approach uses the main features that distinguish signal from atmospheric muon and neutrino backgrounds: clustering around a direction in the sky and harder spectrum (typically $\sim E^{-2}$ spectra are expected for neutrinos directly accelerated in non-relativistic supernova shocks or in relativistic jets). The technique uses scrambled data samples, since the background is uniform in time and hence in right ascension and the signal is assumed to be small compared to backgrounds. Since data and not simulation are used to calculate the background test statistics distribution, the posttrial p-value for the hottest spot in the sky is solid since it comes from the comparison of the data outcome to the scrambled data maps. When unblinded, the $40+59$ string data of IceCube showed nothing incompatible with the atmospheric backgrounds [8]. The skymap of significances is shown in Fig. 4 (left). Similar result has been presented by ANTARES: an update of the published result [21] improved the sensitivity by a factor of 2.5. Fig. 4 (left) shows the average sensitivities and upper limits as a function of declination for various experiments. IceCube limits are not only challenging models for extra-galactic sources, but also most optimistic models for galactic sources. In Fig. 4 (right) we show the case of the Crab Nebula [23], taken as a benchmark to understand where our limits stand with respect to gamma-astronomy observations. Nonetheless, it for sure accelerates photons to tens of $\mathrm{TeV}$. IceCube $90 \% \mathrm{CL}$ limit for the configuration of 40 strings $(1 / 2$ of IceCube) is only a factor of 3.4 higher than the luminosity observed in $\mathrm{TeV}$ photons by H.E.S.S. For the Kappes et al. model the IceCube 90\% CL limit is a factor of 6 higher (references of models are in [23]). This model derives from parent protons the gamma spectrum observed by H.E.S.S. and the muon neutrino one that is reduced by oscillations at Earth by about a factor of two.

In conclusions, these results show that neutrino astronomy has started and that neutrino telescopes are approaching the sensitivity of $\mathrm{TeV}$ gamma-astronomy experiments.

\section{References}

[1] I. Tkachev, Cosmic rays, this conference.

[2] A. Kappes, Mini-review on neutrino telescopes, this conference.

[3] G. Lambard, Indirect searches for Dark Matter with the ANTARES neutrino telescope, this conference

[4] Documentation at http://www.icecube.wisc.edu

[5] D. Dornic for ANTARES Coll., Searches for point sources of high energy cosmic neutrino with the ANTARES telescope, this conf.

[6] A. Tsirigotis, Towards the Very Large Volume Mediterranean Neutrino Telescope, KM3NeT, this conference

[7] R. Abbasi et al., The Design and Performance of IceCube DeepCore [arXiv:1109.6096].

[8] IceCube Coll., The IceCube Neutrino Observatory I: Point Source Searches [arXiv:1111.2741]

[9] A. M. Hillas, Ann. Rev. Astron. Astrophys. 22 (1984) 425.

[10] K. Kotera \& A. Olinto, arXiv:1101.4256. 

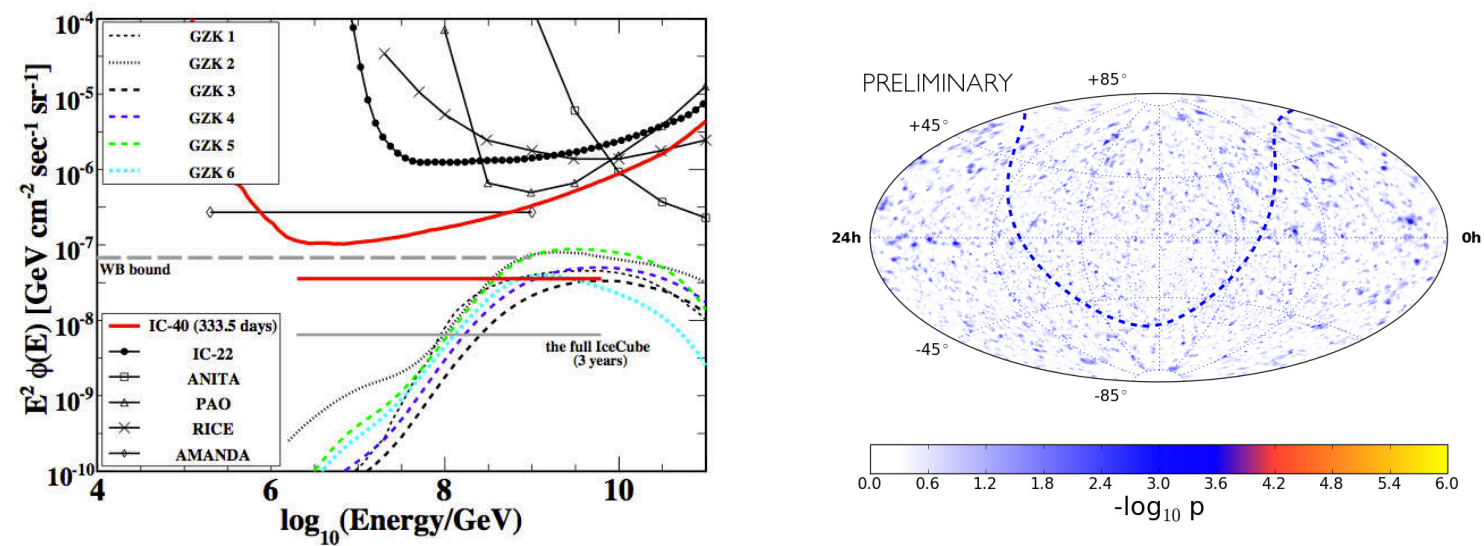

Figure 3: Left: All flavor neutrino flux differential limit (90\% c.l.) and $E^{-2}$ spectrum integrated limit for IceCube (red solid lines) and for $3 \mathrm{yr}$ of the full detector (lowest horizontal line) [17]. The minimum of the differential flux limits indicates the region of maximum sensitivity of the detector. Various model predictions (assuming primary protons) are shown for comparison. The gray dashed horizontal line indicates the W\&B flux bound with cosmological evolution [11]. References are in [17]. Right: The equatorial coordinate hammer-aitoff projection map of the $40+59$ string samples used for IceCube point-source searches. The dashed line represents the Galactic plane.
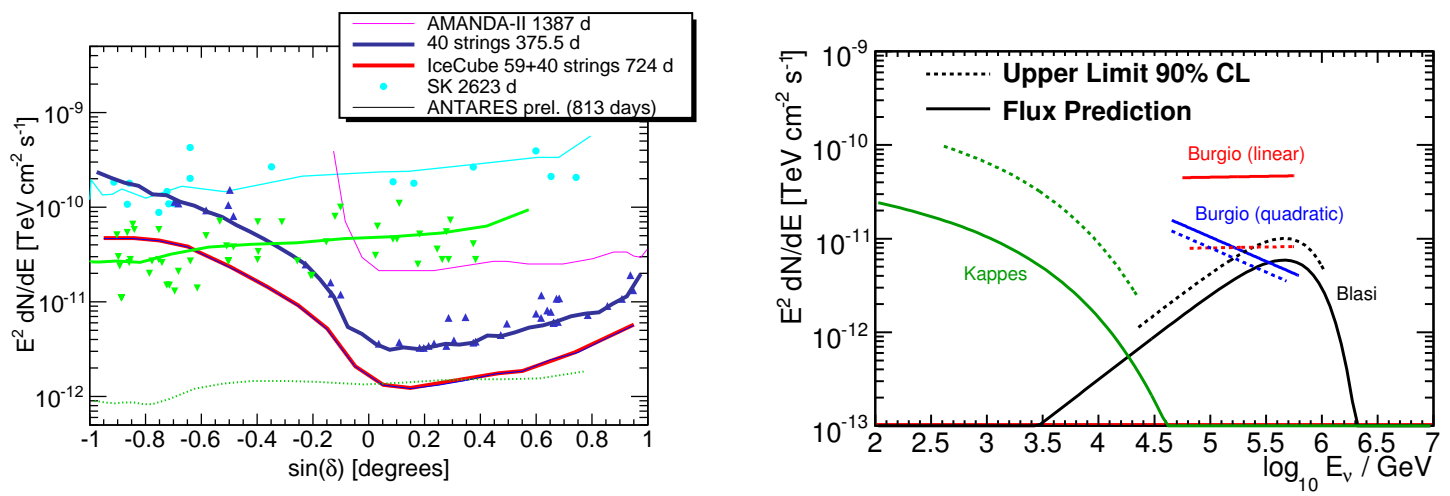

Figure 4: Left: $90 \%$ c.l. sensitivities and upper limits vs declination for an $E^{-2} v$ flux and for various experiments. The curves covering the whole sky are the median sensitivity from top to bottom for: 40 strings of IceCube [19], including upper limits for specific sources (triangles), and merged sample of $40+59$ strings (preliminary) [8]. The recent ANTARES and Super-Kamiokande limits and the KM3NeT sensitivity are shown (references are in [22]). Right: Predicted fluxes from the Crab (solid lines) and 90\% c.l. upper limits (dashed lines) for $1 / 2$ of IceCube (see Ref.s in [23]).

[11] E. Waxman \& J. N. Bahcall, Phys. Rev. D59 (1998) 023002.

[12] E. Waxman, Astronomy at the Frontiers of Science, [arXiv: 1101.1155]

[13] R. Abbasi et al., Phys. Rev. D 84 (2011) 082001

[14] R. Abbasi et al., Phys. Rev. Lett. 106 (2011) 141101.

[15] N. Whitehorn for the IceCube Coll., Results from high-energy neutrino searches from gamma-ray bursts with IceCube, proc. of TAUP2011, Munich. 
[16] D. Guetta et al., Astrop. Phys. 20 (2004) 429.

[17] R. Abbasi et al., Phys. Rev. D83 (2011) 092003.

[18] P. Allison et al, [arXiv:1105.2854].

[19] R. Abbasi et al, Time-Dependent Searches for Point Sources of Neutrinos with the

[20] R. Abbasi et al., ApJ 732 (2011) 18

[21] S. Adri'an-Mart'inez et al., Astrop. J 743 L14

[22] L. Anchordoqui \& T. Montaruli, Ann. Rev. Nucl. Part. Sci. 60 (2010) 129.

[23] R. Abbasi et al., acc. by ApJ [arXiv:1106.3484] 